\title{
Accessibility and Utilization of Emergency Obstetric Care in Rural Settings of Wardha District- Beneficiaries' Perspective
}

\author{
Sarika Dakhode ${ }^{1}$, Abhay Gaidhane ${ }^{2}$, Sonali Choudhari ${ }^{3}$ \\ ${ }^{1}$ Department of Community Medicine, Jawaharlal Nehru Medical College, Datta Meghe Institute of Medical \\ Sciences (Deemed to be University), Wardha, Maharashtra, India. ${ }^{2}$ Department of Community Medicine, \\ Jawaharlal Nehru Medical College, Datta Meghe Institute of Medical Sciences (Deemed to be University), \\ Wardha, Maharashtra, India. ${ }^{3}$ Department of Community Medicine, Jawaharlal Nehru Medical College, Datta \\ Meghe Institute of Medical Sciences (Deemed to be University), Wardha, Maharashtra, India.
}

\section{ABSTRACT}

\section{BACKGROUND}

In India, Emergency Obstetric Care services were started under RCH-II with the goal to reduce MMR to less than 100/lakh live births, and increase institutional deliveries to $80 \%$ by 2010 . Strategy was to enhance availability and access to EmOC, for averting unpredictable death during pregnancy and childbirth. We wanted to determine the perception of beneficiary women about accessibility and utilization pattern of EmOC at peripheral health care facilities.

\section{METHODS}

The present qualitative study was conducted in one of the eight blocks of Wardha (Maharashtra) from May to October-2017. Data was collected by in-depth interview of service users (21 beneficiaries). Notes were transcribed and then translated into English. Respondents' verbatim that are significant and illustrative as per theme of study were used for analysis.

\section{RESULTS}

Facilitators for EmOC were found to be birth preparedness, promotion of institutional delivery, registration for JSY/JSSK through health workers along with provision of some EmOC services. Socio-cultural beliefs, leading to delay in recognition of danger signals to access care, transportation delay due to poor access of ambulance and identified vehicles along with insufficient coverage of JSSK program, were the main barriers. Beneficiaries who required EmOC were dissatisfied due to frequent referral \& travelling, expenditure on transport \& food, loss of daily wages, managing dependent family members and domestic work.

\section{CONCLUSIONS}

Needy women failed to receive the timely and quality EmOC services at periphery due to delayed decision making, inadequate free transport facility from health system, lack of trained staff and sufficient resources to manage difficult cases.
Corresponding Author: Dr. Sonali Choudhari Professor,

Department of Community Medicine, Jawaharlal Nehru Medical College, Datta Meghe Institute of Medical Sciences (Deemed to be University), Wardha, Maharashtra, India.

E-mail: sonalic27@yahoo.com

DOI: $10.14260 /$ jemds/2020/99

Financial or Other Competing Interests: None.

How to Cite This Article:

Dakhode S, Gaidhane A, Choudhari S. Accessibility and utilization of emergency obstetric care in rural settings of Wardha district- beneficiaries' perspective. J. Evolution Med. Dent. Sci. 2020;9 (07):437442, DOI: $10.14260 /$ jemds/2020/99

Submission 30-11-2019,

Peer Review 23-01-2020,

Acceptance 29-01-2020,

Published 17-02-2020.

\section{KEY WORDS}

Emergency Obstetric Care, Utilization Pattern, Qualitative Study 


\section{BACKGROUND}

Globally, about 800 women die every day due to pregnancy and childbirth related preventable causes where India accounts for $20 \%$, and 44,000 deaths are estimated annually in the country.[1] MMR of 174 (2015) $^{[2]}$ and 130/lakh live births in (2016) ${ }^{[3]}$ was below the MDG target for India and hence efforts of $\mathrm{MoH} \& \mathrm{FW}$ is directed towards for achieving the Sustainable Development Goal target that is MMR below 70 by 2030. Since the 1990s, WHO, UNICEF, UNFPA has recognized that Emergency Obstetric Care (EmOC) is one of the cost-effective strategies for reduction of maternal deaths. ${ }^{[4,5]}$ In India, EmOC services were started under RCH-II program to reduce MMR less than 100 and increase institutional deliveries to at least $80 \%$ by 2010.[6,7] Implemented strategy was to enhance availability of facilities and increase access to EmOC. EmOC has two types of services ${ }^{[5]}$ Basic EmOC includes six medical interventions (signal functions) such as parenteral administration of antibiotics, anticonvulsants, oxytocics; assisted vaginal delivery, manual removal of placenta and removal of retained products of conception. Comprehensive EmOC includes caesarean section and blood transfusion in addition to basic EmOC.

Carrying out of well-focused lifesaving EmOC require effectively functioning health system. This includes clean delivery supplies, essential medicines and equipment along with presence of personnel qualified to provide it. On the other hand, merely operationalizing facility by providing skilled manpower and equipment does not ensure accessibility and optimum utilization of service by needful beneficiaries. Knowledge and faith of people about location, content and quality of care offered by the facilities (meant for the purpose) are important factors determining extent of utilization of such facilities. Beneficiaries' view too is essential to understand the status or pattern of accessing EmOC in the district. Keeping in view the importance of EmOC to avert unpredictable women's death during pregnancy and childbirth, the present study is carried out with an objective to assess the perspective of beneficiary women about accessibility and utilization pattern of EmOC at peripheral health care facilities.

\section{METHODS}

This qualitative study was conducted in one of the blocks of Wardha District (Maharashtra) from May to October 2017. Selected block has five Primary Health Care centres (PHC) and two Rural Hospitals (RH) which were included in study. Accessibility and utilization of EmOC was studied through beneficiaries' perspective using qualitative method. Study participants were women who availed EmOC services from public health care facility of the identified block or who got referred further to higher facility for availing EmOC in recent six months. In depth interviews were conducted to get the views of women. Interview guide was prepared for facilitating discussion among beneficiaries, which included the following issues:

- ANC services received in recent last pregnancy.
- Knowledge about the danger signs and birth preparedness.

- Reaching the health care facility, care received and referral pattern.

- Satisfaction for services and suggestions to improve services.

Assistance from grass-root level health workers like ASHA worker and ANM of public health facility was sought for selection of beneficiary and to conduct interviews. Three beneficiaries were selected from each facility area and data collection done till reaching the saturation point. Women who were responsive and conveniently available included in the study sample. Interviews were conducted in local language (Marathi); Confidentiality was assured by taking written informed consent. During discussion, the authors tracked the events retrospectively from the time of medical emergency till the utilization of services; like what health facility did they approach during emergency; health care service received; how referral occurred and where was the delay (if any), amount of out of pocket expenditure the beneficiary/family bore and the outcome. Interviews were audio taped. Overall, each interview lasted for sixty minutes. Thus around 21 women were included in the sample, considering the data saturation point

\section{Data Analysis}

Notes were transcribed and then translated into English. Respondents' verbatim that were significant and illustrative as per theme of study were used for analysis. These transcripts were coded using descriptive words and phrases, organized into the concern subject and themes that emerged were outlined. Qualitative data is presented in text form with quotes to illustrate study findings and is organized under suitable headings and labels in accordance with the research objectives.

\section{RESULTS}

Pattern of seeking EmOC care by women was studied by tracking events retrospectively from time of emergency till time of receiving care at any of the health facility.

\section{Decision Making (First Delay)}

To receive the timely and efficient emergency care during pregnancy and childbirth, it is important for women to be aware of and recognize the danger signs, which can facilitate decision making. The interviews revealed pregnant women were informed through health workers (ASHA and ANM) about signals of labour and birth preparedness; but they didn't correctly interpret or perceive it as problem; as a result many didn't seek timely care. One of the women who availed the services narrated..... "...I was in $8^{\text {th }}$ month of pregnancy.... had some pain. I thought it is routine pain so did not tell anybody. The pain did not reduce and became worse next day, so I told ASHA. She (ASHA) told me this may be serious, and we rush to PHC in evening, where the nurse examine me and refer me to district hospital where I was given some tablets and saline (IV fluids). They kept me in ward for seven days and then discharged. It was my fault. I did not realize 
that little pain in eighth month may be serious." It was also noted in discussion that socio-cultural beliefs and family system influences the care seeking behaviour. A primigravida woman narrated her encounter with her mother-in- law. "...My mother-in-law argued that I should not report to the hospital when I started having abdominal pains...that...it was too early to go and as a result we may stay at the hospital for a long time before I deliver...she gave me some herbal medicine.....she believed the herbs would be useful in making the delivery quick and less painful. My pain did not relieved, so I was rushed to the PHC and then to hospital where I delivered by operation (LSCS)"

\section{Transportation (Second Delay)}

The common mode of transportation in remote villages under the jurisdiction of studied PHC were auto-rickshaw, bullock cart or in some instances taxi \& other four-wheeler. All beneficiaries had received information about 'Janani Shishu Suraksha Karyakram' (JSSK) ${ }^{[8]}$ and 'Janani Suraksha Yojana' (JSY) ${ }^{[9]}$ through ASHA and ANM. Out of 21 beneficiaries, 12 were eligible and enrolled for JSY and received incentives but according to them amount was insufficient for the obstetric treatment as they had to spend more at district place. Though ambulance at PHC headquarter was available, women mentioned that they didn't have access to it during emergency in remote villages. Ambulance facility was expected at PHC, but sometimes it was not available as taken out for immunization session in another village, drug procurement at the district, for other referred patient or supervisory visit by PHC Medical Officer (MO). ASHA worker and ANM could help for emergency transport to some extent. Out of 21 women interviewed, nine mentioned that they reached the facility by autorickshaw, four by bullock cart as it was night and no other facility was available, two each by the tractor and sumo (four-wheeler). Four women availed the ambulance services to reach the PHC/RH under the JSSK program and received free treatment completely, while other women had to spend money on one or other area such as transport, diet or medicines.

\section{Referral Pattern (Third Delay)}

Health care delivery system in India is to provide basic EmOC at PHC and comprehensive EmOC at RH and District level under the RCH program.[5] So blood transfusion can't be expected at PHC hence patient is referred to RH or district hospital. However, if transfusion would not be received even at higher facility; patient may suffer due to second and third delay. A woman suffering from bleeding per vaginum was in need of blood transfusion; first she was referred from PHC to $\mathrm{RH}$ and then to district hospital where she didn't receive transfusion and was again referred further to tertiary care center in the district. She Expressed that "...of course there is no blood facility in PHC, but in RH, I was expecting it.....but they send me to Wardha (district hospital) and there too.....sister (nurse) informed us about non-availability of blood of group I belong to..., there is danger to baby's and mother's life...here delivery is not possible...have to go Sewagram hospital (tertiary care hospital)...Expenditure in my delivery was more than expected (expressed sadly)....". Other woman suffering from pregnancy induced hypertension was referred to tertiary centre where caesarean section was conducted. Delivery expenses were more for women who were referred from district hospital to tertiary centre and least for the woman managed at peripheral level itself. One of the beneficiaries suffered during night hours, due to non-availability of MO at PHC. She narrated her experience- "My family took me to PHC at night...but the nurses informed that the health condition of mine cannot be managed there......doctor was not there,.....nurse further added that there is a single MO and he had gone for some training to district health office...ultimately my family had to take me to the main hospital. We would have saved money on transportation if we would have directly gone to district hospital". Under basic EmOC, assisted vaginal delivery can be performed at PHC level by trained MO. In studied area, four interviewed women needed assisted vaginal delivery and episiotomy done by $\mathrm{MO}$ and mild postpartum haemorrhage of other women was also controlled by PHCs doctors.

\section{Expenditure on Accessing Care}

These interviews revealed how lack of money influences the health seeking behaviour of pregnant women and those in labour. In most of the cases, the family members didn't have enough money to be able to pay for transport and/or emergencies as well as extra money for food, while the patient at the hospital. The only alternative for them was to reach out to other people in the village for help, or mortgaging or sell some property or livestock, which contributed to delay in seeking health care. One of the interviewees who availed services in the recent six months narrated her frustration for arranging money. "... I suddenly got severe pain in my $8^{\text {th }}$ month and my husband decided to take me to the hospital... we did not have enough money at that time...My husband had to mortgage our bullock cart to Sahukar....also borrow some money from a neighbour that night; which was not enough...it took him two hours to raise this amount... also he (husband) literally beg the auto rickshaw driver to assist us to reach the facility". One women shared her feelings of sufferings- "I had to stay at district hospital for five to seven days for my treatment of bleeding after delivery......on one side during pregnancy ASHA used to tell us to get the delivery done at government clinic of taluka....but see what happened .....hospital people referred me to district hospital.........my husband lost his labour work, my elder son of three years had to stay at neighbour's home.....it was very hectic to manage home and hospital....then what about tiffin (meal)....who will do it, if my mother-in-law had to stay with me?...that is too much difficult for we rural people.....to and from from city to village..."

\section{DISCUSSION}

For successful outcome of any program or service, one of the key components is the utilization of available services by the needful beneficiary. This depends on provision, accessibility (physical, geographically and social) and acceptability (social, cultural and behavioural). ${ }^{10,11}$ Awareness and recognition of an obstetric complication among pregnant women and within their communities is the first step in initiating appropriate and timely referrals and reducing the decision-making time to seek care. 


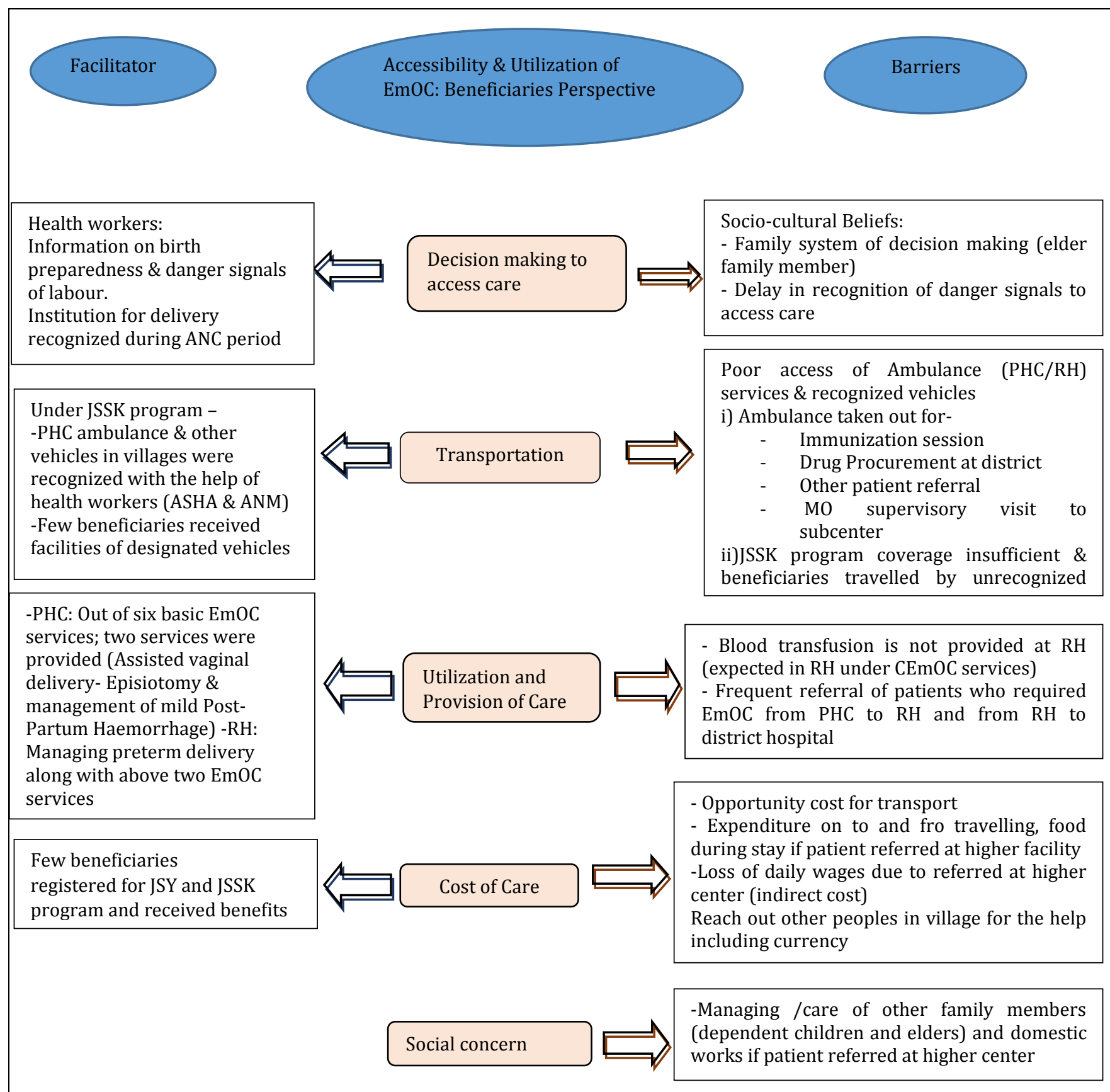

Figure 1. Thematic Framework of Accessibility and Utilization of EmOC in Rural Settings: Beneficiaries

In studied villages, beneficiaries were informed about birth preparedness; and institution for delivery was recognized. In rural India, the decision to seek early delivery care at health centre is usually influenced by either mother or mothers-in-law. Visiting the hospital early in labour was viewed by most of the close relatives as unnecessary; they would prefer to use some home remedies or traditional medicines for problems during pregnancy while waiting for established labour. Awareness of danger signs during pregnancy, delivery or postpartum was low among interviewed women. For creating awareness; mere health education to pregnant women as a part of antenatal care is quite insufficient to get oriented about the emergency. Improvement of community awareness of danger signs and services available at the facilities, including community mobilization for arrangement of transport are critical for improving early access to care and utilization of services. Pregnancy and childbirth are often considered as a natural process; hence, signs and symptoms of complications are seldom recognized as reasons for concern. Thus, ineffective and delay in decision-making at the family level was identified as a barrier in accessing timely EmOC in the present study. In India, decision to seek care (first delay) is most common delay which is influenced by many factors such as, socio cultural beliefs, elderly family members viewpoint, poverty, fear of health system, past experiences and gender inequity.[12,13,14]

A total of 22 cases of maternal deaths were reviewed in a district of Madhya Pradesh (India) and found that all women had tried to access health care. Socio cultural factors such as difficulty in recognition of the complications by family members, gender inequity, and perceptions of low-quality 
health services on the basis of past experiences had contributed to first delay of decisions to seek care.[15] In southern Odisha, making aware to pregnant women and family members regarding danger signals of complications, screening for high risk ANCs by specialist was observed to be lacking during ANC visits.[16] After decision to seek care, timely reaching the facility assists to receive the EmOC. The mode of transport to the hospital is a fundamental determinant in health seeking. This often presented a threat to the survival of women and newborns in developing nations. Even if the women are aware of the danger signs and willing to reach hospital for early care, availability of transportation and road infrastructure facility are crucial to reach the facility on time. Improving physical access has been the primary strategy for increasing health services utilization in developing countries; whereas access to transportation facility for remote villages in studied area was challenging.

In Maharashtra, beneficiaries commonly perceive low quality delivery services in rural settings. Though patient wanted to seek care at FRU or higher facility but waste of time and money in arranging transportation, and care seeking at multiple facilities due to frequent referral leads to delayed reaching appropriate health facilities (second delay).[15,17] Effective functioning referral systems are vital to the accessibility of services. There is only one ambulance available at PHC or RH area, which is highly inadequate for services in 20-25 villages. It is difficult to make available always in remote villages. Hence local autorickshaw is the option in emergency and the last is bullock cart in he studied area. There is need to scale up of the 108 or 102 ambulance services in the Wardha district to reach the remote villages. Simulating partnership between rural communities and local transport owners could be one key strategy in overcoming transport issues during emergency. This scheme was implemented in northwest Nigeria and had led to significant diminution in transportation cost and maternal, perinatal mortality.[18]

Basic EmOC services cover the management of prolonged labour, breech delivery, management of incomplete abortion at PHC and caesarean section and blood transfusion at RH. Interviewed beneficiaries having complications like 'bleeding per vaginum', 'leaking per vaginum', 'raised blood pressure' and 'need of caesarean section due to breech presentation' were referred at district hospital. Some of them were further referred to tertiary care hospital in district. The healthcare providers in same area expressed various reasons for referral such as less manpower at the peripheral center; difficult to manage by MO if clinical conditions get worsened and for the sake of patient's health/benefit.[19] Gupta, et al[20] (2016) studied about referred obstetric cases for appropriateness, timeliness and outcome, observed inadequate antenatal and intranatal care at the periphery level and suggested availability of EmOC in terms of skilled birth attendants, round the clock blood transfusion, need of specialist in obstetric and anaesthetics to reduce maternal mortality. In present study, noted that women often go hopping from one facility to other to receive desired services which often lead to delay in receiving emergency services. Improvements in the quality of service can further boost service utilization. Tej Ram Jat et al[15] observed delayed in receiving adequate care after reaching a health facility because of unavailability of basic and comprehensive EmOC at primary and secondary/tertiary level respectively and lack of motivation in staff.

Banu Morsheda, et al (2011) ${ }^{[21]}$ explored factors related to performance of delivery centres by qualitative methods. Facilitators of center performance were quality work done by community health workers, geographical access, free services and cleanliness. Barriers were lack of health workers, less motivation, frequent dropout due to low remuneration, and recurrent slum demolition. Beneficiaries were hesitating to avail obstetric services at these centres due to lack of comprehensive services and chances of referral to other hospital which might compel them for caesarean. A review identified some of the factors such as; lack of skills and confidence of health providers at facility creates tendency to pass the patent to higher centers rather than providing designated services under EmOC. There is need of clinical guidelines, communication and supervision for referral.[22] Cost of receiving care is a key factor in determining the choice of place of delivery during an emergency, and it contributes to the first delay in seeking care outside home.[23] The cost of maternity care and the inability to meet the cost is a significant barrier to EmOC access, thus an important determinant of maternal and perinatal mortality.[24] Out of pocket expenditure will be more in case of referral. Although maternity care is officially provided free of charge in public hospitals in Wardha, due to frequent referral indirect and/or opportunity costs still remain a substantial obstacle in timely access to obstetric care in rural settings. Women's access to EmOC is further compromised if the care being sought is going to be very costly. The distance and time involved in seeking health care often determine how much cost a family may incur.

Janani Shishu Suraksha Karyakram ${ }^{8]}$ is functioning at district hospital but beneficiaries were unsatisfied due to poor coverage. Cash incentive program 'Janani Suraksha Yojana' [9] may be helpful to some extent but it doesn't replace the total economic expenditure or loss on received care by the suffered family. However, designated EmOC services if are not provided at the PHC or RH level to the beneficiary then women seldom get referred to higher level. There was increased burden of the expenditure on transportation and food during stay of accompanying family members in the hospital. Managing domestic work, loss of daily wages, and care of other dependent family members were the common issues for the suffered family. If she is referred to the private institute along with indirect cost, the delivery expenses (direct cost) will be increased than expected.

\section{CONCLUSIONS}

Most of the beneficiaries were (observed to be) dissatisfied when they came to know that required obstetric service was not going to be provided at PHC or RH and even at district hospital. Very few beneficiaries were provided facility ambulance when referred further to higher center. Needy women failed to access and receive the timely and quality EmOC services at periphery due to delayed decision making, inadequate free transport facility from health system, lack of trained staff and sufficient resource especially blood transfusion to manage difficult cases, and fear of further 
worsening of case with obstetric emergency on the part of health care providers.

\section{ACKNOWLEDGEMENT}

We would like to thank Civil Surgeon, District Health Officer, Additional District Health Officer of Wardha District for permission to conduct the study in study block.

\section{REFERENCES}

[1] Maternal Health in Focus. Maternal Health UNICEF, India. http://unicef.in/Whatwedo/1/Maternal-Health

[2] Maternal mortality in 1990-2015, Maternal Mortality Estimation Inter-Agency Group. WHO, UNICEF, UNFPA, World Bank Group, and United Nations Population Division. WHO 2016. https://www.who.int/gho/maternal_health/countries/i nd.pdf

[3] Poonam KS. India has achieved groundbreaking success in reducing maternal mortality. Regional Director, WHO, South East Asia Regional office, 2019. http://www.searo.who.int/mediacentre/features/2018 /india-groundbreaking-sucess-reducing-maternalmortality-rate/en/

[4] World Health Organization, World Health Report, 2005: make every mother and child count. https://books.google.co.in/books?isbn=9241562900 [Accessed on 30/06/17]

[5] WHO, UNFPA, UNICEF. Guidelines for monitoring the availability and use of obstetric services. 2nd edn. UNICEF, New York, Aug 1997. www.unicef.org

[6] National Commission on Macroeconomics and Health (New Delhi) Ministry of Health \& Family Welfare, Government of India. NCMH Background Papers-Burden of Disease in India. September, 2005. http://www.who.int/macrohealth/action/NCMH_Burde n\%20of\%20disease_ _ (29\%20Sep\%202005).pdf [Accessed on: 17/07/13]

[7] Govt. of India: Ministry of health and family welfare: Project implementation Plans, RCH II Program: RCH II document 1, Policy framework: December 2004: p. 1-58.

[8] Maternal Health Division, Ministry of Health and Family Welfare. Government of India. NRHM Guidelines for Janani-Shishu Suraksha Karyakram, 2011. https://www.nhp.gov.in/janani-shishu-surakshakaryakaram-jssk_pg

[9] Ministry of Health \& Family Welfare, Government of India. NRHM Guidelines for Janani Suraksha Yojana (JSY), 2005. http://documents.gov.in/OR/2384.pdf
[10] Rao PS, Richard J. Socio-economic and demographic correlates of medical care and health practices. J Biosoc Sci 1984;16 (3):343-55.

[11] Rohde J, Viswanathan H. The rural private practitioner. New York: Oxford University Press 1995.

[12] Murthy BK, Murthy MB, Prabhu PM. Maternal mortality in a tertiary care hospital: a 10-year review. Int J Preventive Med 2013;4 (1):105-9.

[13] Paul B, Mohapatra B, Kar K. Maternal deaths in a tertiary health care centre of Odisha: an in-depth study supplemented by verbal autopsy. Indian J Comm Med 2011;36 (3):213-6.

[14] Khandale SN, Kedar K. Analysis of maternal mortality: a retrospective study at tertiary care centre. Int J Reprod Contracept Obstet Gynecol 2017;6 (4):1610-13.

[15] Tej RJ, Prakash RD, Isabel G, et al. Socio-cultural and service delivery dimensions of maternal mortality in rural central India: a qualitative exploration using a human rights lens. Glob Health Action 2015;8 (1):24976.

[16] Mahapatro M. Equity in utilization of health care services: perspective of pregnant women in southern Odisha, India. Indian J Med Res 2015;142 (2):183-9.

[17] Ghumare JP, Padvi NV. Assessment of maternal deaths using three delay model at a tertiary care centre in rural Maharashtra, India: retrospective six years study. Int J Reprod, Contracept, Obstet \& Gynecol 2018;7 (8):30437.

[18] Shehu D, Ikeh AT, Kuna MJ. Mobilizing transport for obstetric emergencies in North-west Nigeria. The Sokoto PMM Team. International Journal of Gynecology \& Obstetrics 1997;59 (Suppl 2):S173-80.

[19] Uttamrao DS, Abhay G, Pramita M, et al. Health Workforce related challenges for Emergency Obstetric Care at peripheral health facilities: providers' perspective. J Holist Nurs Midwifery 2018;28 (1):44-55.

[20] Rohit GP, Nitin CS, Vasantrao GN. Maternal and fetal outcome in referred patients to tertiary care center. Sch J App Med Sci 2016;4 (5C):1624-31.

[21] Banu M, Hashima-E-Nasreen, Nahar S. Factors affecting the performance of delivery centre. Working Paper No. 25, BRAC, Aug 2011.

[22] Singh S, Doyle P, Campbell OM, et al. Referrals between public sector health institutions for women with obstetric high risk, complications or emergencies in India - a systematic review. PLoS One 2016;11 (8):e0159793.

[23] Hildah E, Samuel M, Jean-Christophe F. Barriers to Formal Emergency Obstetric Care Services' Utilization. J Urban Health 2011;88 (Suppl 2):356-69.

[24] Census of India 2011. Rural Urban Distribution of Population, India. http://www.censusindia.gov.in 